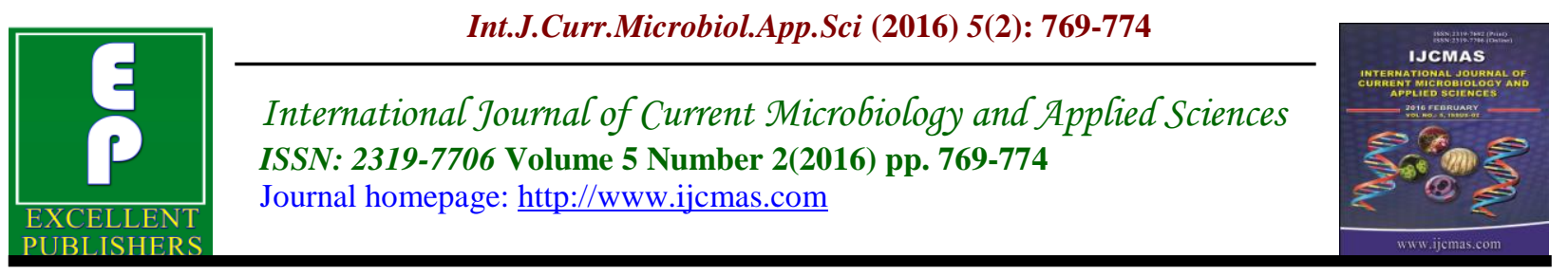

Original Research Article

doi: http://dx.doi.org/10.20546/ijcmas.2016.502.087

\title{
Typing and Growth Characteristics of Salmonella paratyphi A obtained from Blood sample of a Paratyphoid Case
}

\author{
Arti Bharmoria ${ }^{1 *}$, Shahana Majumdar ${ }^{1}$ and Vipin Behari Vaish ${ }^{2}$ \\ ${ }^{1}$ Sharda University, Greater Noida, U.P., India \\ ${ }^{2}$ National Salmonella and Escherichia Centre, Central Research Institute, Kasauli, H.P., India \\ *Corresponding author
}

\begin{abstract}
A B S T R A C T
Keywords

Paratyphoid fever has been a disease of public health problem in Asian countries. In India clinical cases of paratyphoid had been emerging on escalating rates. This problem requires a consistent monitoring of the isolation throughout the nation to

Biotyping; Serotyping; Cultivation; Salmonella paratyphi $\mathrm{A}$; India. analyze the spectrum of the Salmonella enteric serovar Paratyphi A. This study examined isolation of Salmonella paratyphi A from a clinical sample of paratyphi A obtained from Yashoda hospital, Ghaziabad. This sample was characterized by biotyping as well as serotyping. The characterization of the bacteria was reconfirmed at National Salmonella and Esherichia Centre, Kasauli. The isolate serotyping was based on somatic $O$ and phase $1 / 2$ flagellar antigens by agglutination tests with antisera according to the Kauffmann White scheme. The clinical isolate was confirmed to be a case of Salmonella paratyphi A by biotyping

Article Info

Accepted:

28 January 2016

Available Online:

10, February 2016 and serotyping. The bacteria achieved a complete growth when propagated in the laboratory in controlled temperature, humidity and $\mathrm{pH}$. This study enlights the Salmonella paratyphi A characterization by biotyping and serotyping as well as cultivation tecnhnique in laboratory. The escalating rate of paratyphoid infections appears as a budding problem which requires the advancement in diagnostic techniques as well as effective vaccines for Salmonella enterica serovar Paratyphi A to deal with emerging cases of infections.
\end{abstract}

\section{Introduction}

Enteric fever, includes typhoid fever caused by Salmonella typhi and paratyphoid fever caused by $S$. enterica serovar Paratyphi A and is one of the most important febrile illnesses in tropical and subtropical countries, with high rates of morbidity and mortality. S. paratyphi A can be isolated from the blood and feces from paratyphoid fever patients $(1,2)$. In India, however Salmonella enterica serotype Typhi remains predominating in causing enteric fever, isolation of Salmonella enterica serotype Paratyphi A causing the same disease, has also been reported increasingly in various parts of North and South India including regions like New Delhi, Chandigarh, Shimla, Kolkatta, Rourkela, Orissa, Calicut and Manipal (3,4,5,6,7,8,9,14).

Typhoid fever is estimated to have caused 
21.6 million illnesses and 216,500 deaths globally in 2000, and based on one case of paratyphoid fever for every four cases of typhoid fever, $S$. paratyphi A is estimated to have caused an additional 5.4 million illnesses in 2000. These estimates, however, are known to vary widely between regions while the cases of enteric fever caused by $S$. paratyphi A are still increasing (16). The incidence of typhoid and paratyphoid fever is increasing especially in South Asia and South Africa, where the annual incidence is $622 / 100,000$ and 233/100,000, respectively (17).

S. paratyphi A has the flagellar antigens and is a member of somatic $\mathrm{O}$ group $\mathrm{A}$ with more prevalent phage type I. For serotyping of Salmonella, lipopolysaccharide epitopes in bacterial membrane ( $\mathrm{O}$ antigens) and flagella proteins ( $\mathrm{H}$ antigens) should be identified with the respective antibodies (15). Biochemically, the organism resembled $S$. enterica serotype Typhi; particularly having negative citrate, arginine, ornithine, arabinose, and rhamnose reactions. However the identity of both organisms can be confirmed by full serotyping via Kauffman-White scheme, in which the $S$. enterica serotype Paratyphi A isolate is assigned the antigenic formula I 2,12:a:1, whereas the $S$. enterica serotype Typhi isolate is assigned the antigenic formula I 9,12[Vi]:d:_(18). The given study is exploring the biochemical and serotyping characteristics of Salmonella paratyphi A isolate obtained from a clinical paratyphoid case with emphasis on analysis of growth characteristics of the isolated bacteria.

\section{Materials and Methods}

For the biotyping, all media and biochemicals were obtained from Hi Media Lab. Pvt. Ltd., India. The biotyping of isolate $S$. paratyphi A was performed by conventional biochemical tests $(10,11)$ and confirmed by serotyping on the basis of somatic $\mathrm{O}$ and phase 1 and phase 2 flagellar antigens by agglutination test using standard Salmonella agglutinating factor sera in accordance to the scheme of KauffmannWhite $(12,13)$. The isolates were cultured on nutrient agar, Mac-conkey agar and blood agar plates.

The sera raising was performed in rabbits by following an immunization schedule with $0.25 \%$ formalin killed $S$. paratyphi A suspension of $0.5 \mathrm{ml}, 1.0 \mathrm{ml}, 1.5 \mathrm{ml}, 2.0 \mathrm{ml}$ dose by subcutaneous route on day 1,2,7 and 11. The animals were test bled after one week of the last injection and tested for homologous titres by agglutination test. The titre of $\geq 1: 1000$ for $O$ sera and $\geq 1: 1200$ for $\mathrm{H}$ sera was the satisfactory level for testing purpose. The animals were bled by cardiac bleeding method. Blood was collected in dry sterile test tubes and then stored in a refrigerator $\left(+4^{\circ} \mathrm{C}\right)$ overnight. The blood clot was removed by centrifugation and all the sera from different bleedings were pooled together and filtered by $0.45 \mu \mathrm{m}$ micro-filter.

For serotyping purpose an 18 hour broth culture of the clinical isolate was taken. It was then $0.25 \%$ formalinized and kept at room temperature for 30 minutes. The culture was centrifuged at 2,000 rpm for 15 minutes. The supernatant was discarded and the deposit was used for serotyping work. 45 drops of the centrifuged deposit were placed on a clean glass slide. To the first drop was applied a drop of $\mathrm{H}$ : a factor antisera. The agglutination was positive, the Salmonella serotype was identified. The tube agglutination test was also performed respectively. These confirmatory tests were performed by using standard diagnostic antigen and factor antisera for Salmonella paratyphi A. 
For the serological specificity the bacterial culture of $S$. paratyphi A was grown on solid soyabean casein digest (SCD) media. The antiserum against Salmonella paratyphi $A$ was added to the media plates with bacterial growth and these plates were then incubated at $37^{\circ} \mathrm{C}$ for overnight.

The studies for analysis of growth pattern analysis were also carried out $(20,21)$. A fresh colony of bacteria was inoculated into SCD media. $2.5 \mathrm{ml}$ of the turbid growth was inoculated to $50 \mathrm{ml}$ of SCD media and the flask was incubated at $37^{0} \mathrm{C}$ for overnight. $7.5 \mathrm{ml}$ of the turbid growth obtained was inoculated to $150 \mathrm{ml}$ of SCD media and the flask was incubated at $37^{\circ} \mathrm{C}$ for overnight at $\mathrm{pH}$ 7. Next day $50 \mathrm{ml}$ of the turbid growth was inoculated to 4 flasks having 1.5 lts SCD media in each flask and these flasks were incubated at $37^{\circ} \mathrm{C} / 125 \mathrm{rpm}$ on rotary shaker. After 3 hours the turbidity appeared in the flasks. $40 \%$ sterile glucose was added to make final concentration of $5 \%$ glucose in each flask.

Added $2 \mathrm{~N}-\mathrm{NH}_{4} \mathrm{OH}$ to make the $\mathrm{pH} 7.2$ and again incubated them at $37^{\circ} \mathrm{C} / 125 \mathrm{rpm}$ on rotary shaker. The culture was grown in flasks at $37^{\circ} \mathrm{C}$ on rotary shaker for 12 hours. Kept the $\mathrm{pH}$ at 7.00 using $2 \mathrm{~N}-\mathrm{NH}_{4} \mathrm{OH}$. The culture was kept at $37^{\circ} \mathrm{C}$ for overnight incubation. Repeated Gram staining was performed to check the purity of culture at every stage. The bacterial growth was found pure and uniform. The bacterial samples were withdrawn to analyze the absorbance at $600 \mathrm{~nm}$ (19). The $\mathrm{pH}$ was adjusted to 7.2 using $2 \mathrm{~N}-\mathrm{NH}_{4} \mathrm{OH}$ and formalin at the final concentration of $1 \%$ added for final inactivation. The culture was kept for inactivation at room temperature for 24 hours. This Salmonella paratyphi A was further used for the harvesting of lipopolysaccharide for the O-specific polysaccharides.

\section{Results and Discussion}

S. paratyphi A was sub-cultured on blood agar and Mac-Conkey agar, after overnight incubation at $37^{\circ} \mathrm{C}$, blood agar showed non pigmented (grey-white) colonies of size 1-2 mm, opaque, non-hemolytic, moist, circular with a smooth convex surface and the entire edge. The growth on MacConkey agar consisted of non lactose fermenting colonies of similar morphology. Colonies were catalase positive and oxidase negative. Gram stained smear from the growth revealed Gram-negative bacilli, $2-4 \mu \mathrm{m} \times 0.6 \mu \mathrm{m}$ in size, non-capsulate and non-sporing. The bacilli were motile.

Biotyping confirms Salmonella paratyphi A by cultures which were Lactose negative, Catalase positive, Oxidase negative, Indole negative, Methyl Red positive, Vogus Proskaur negative, Citrate negative, Urease negative, Triple Sugar Iron showed K/A glucose acid with gas, Nitrate reductase positive and Lysine negative.

The positive agglutination of Salmonella paratyphi A antisera with Salmonella paratyphi A bacteria was observed. The colonies showed serological specificity by creating haloes of immune-precipitation when grown on SCD agar media containing antiserum against Salmonella paratyphi A.

Absorbance studies were conducted to analyze the growth pattern of the Salmonella paratyphi $A$. The samples of bacterial growth were collected at various steps during propagation of bacteria and the optical density at $600 \mathrm{~nm}$ was checked. The results are given in Table no.-01 and in Diagram No.-01.

There was an up-scaling in the absorbance at $600 \mathrm{~nm}$ of the bacteria. As the volume of media increases the O.D of the culture was found in increasing order. The samples were 
obtained at 12 hours of growth and the culture was in the log phase. This indicates that the Salmonella paratyphi $A$ seed was propagated with better culturist behavior.

Table.1 Growth Analysis of Salmonella paratyphi A

\begin{tabular}{|c|c|c|c|c|}
\hline S.No. & Volume of Media & Incubation time & $\begin{array}{c}\text { Purity } \\
\text { (Gram Staining) }\end{array}$ & OD at 600nm \\
\hline 1. & $2 \mathrm{ml}$ & Nil & Pure & 0.201 (Autozero) \\
\hline 2. & $20 \mathrm{ml}$ & 12 hour & Pure, Uniform & 1.375 \\
\hline 3. & $50 \mathrm{ml}$ & 12 hour & Pure, Uniform & 1.215 \\
\hline 4. & $100 \mathrm{ml}$ & 12 hour & Pure, Uniform & 1.555 \\
\hline 5. & $3000 \mathrm{ml}$ & 12 hour & Pure, Uniform & 2.398 \\
\hline
\end{tabular}

Diagram No.-01: Growth analys is of Salmonella paratyphi A

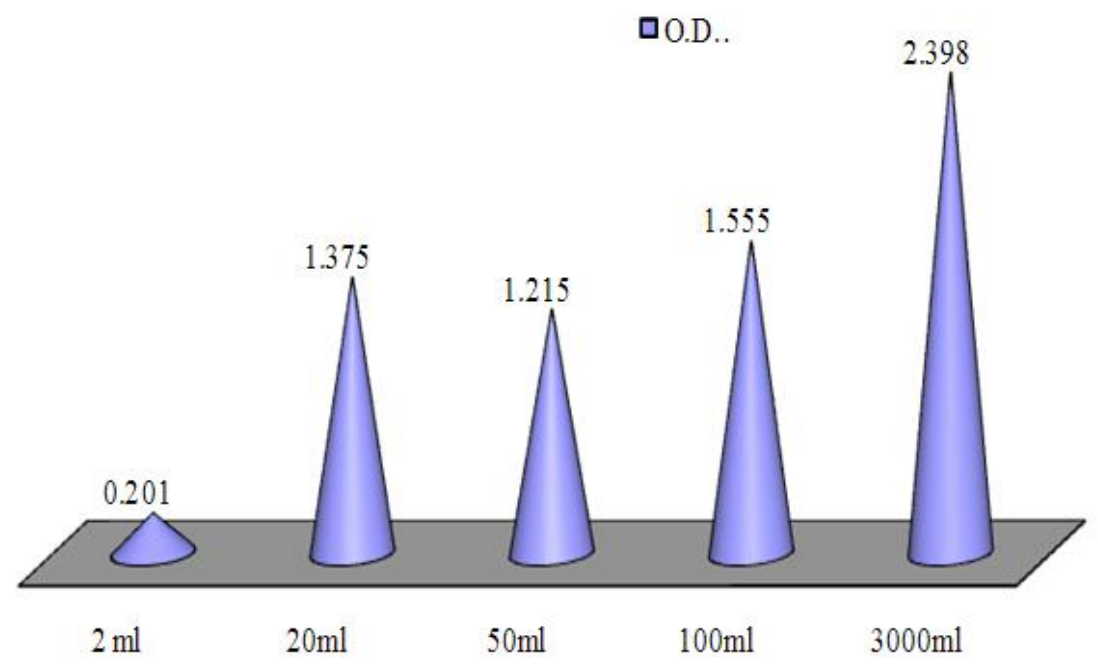

The 'gold standard' for identifying the cause of an infection is the isolation and identification of the causative agent of disease. In the absence of a viable bacterium, antibody tests can give evidence of infection provided that suitable immunoassays, based on well-characterized antigens, are used. Biotyping characters are considered as markers of major importance in the typing of salmonellae. On the otherhand, serotyping is based on the long standing observation that the microorganisms from the same species can differ in the antigenic determinants expressed on the cell surface. Serotyping method is used to differentiate isolates of Salmonella beyond the subspecies level. The reference sera used for serotyping contain antibodies to the panel of the lipopolysaccharide and flagellar antigens of $S$. paratyphi A. The $S$. paratyphi A bacteria when cultivated at temperature of $37^{\circ} \mathrm{C}$ at $\mathrm{pH}$ of 7.2-7.4 in presence of additional glucose ratio then the log phase can be achieved within 12 hours. Higher volumes of media lead to better bacterial yields in comparison to lower volumes of media.

The present study endorses the observation of Salmonella paratyphi A as rapidly 
emerging pathogen of enteric fever. So, continued surveillance on the mutual analysis by biotyping and serotyping of Salmonella serovar paratyphi $A$, the multidrug resistance, diagnosis and antimicrobial susceptibility from different regions of the country will help in up-dating the knowledge for proper therapeutic cure and vaccine development.

\section{Acknowledgement}

Authors would sincerely acknowledge with special thanks for the contribution of Dr. S.P. Garg (Ms. Bio-Med Pvt. Limited, Ghaziabad) for sponsoring and facilitating this study at their laboratories. The authors would also like to thank all the staff members National Salmonella and Escherichia Centre, Central Research Institute, Kasauli, India for providing their assistance and support for accomplishment of this study.

\section{References}

1. Teh CSJ, Chua KH, Thong KL Cindy SJT, Kek HC, Kwai LT (2014) Paratyphoid Fever: Splicing the Global Analyses. International Journal of Medical Sciences, 11(7): 732-741. doi: 10.7150/ijms.7768

2. Priyamvada R, Deepti R, Sonia M (2015) A case of extended spectrum beta-lactamase producing Salmonella enterica serotype Paratyphi A from India. Indian Journal of Pathology and Microbiology, 58 (1): 113-114

3. Sarika J, Tulsi DC, (2013) Antimicrobial resistance among blood culture isolates of Salmonella enterica in New Delhi J Infect Dev Ctries 7(11):788-795.

4. Shanta D, Surojit D, Utpala M, Priyanka J, Indranil R, et al. (2014) Antimicrobial Resistance, Virulence
Profiles and Molecular Subtypes of Salmonella enterica Serovars Typhi and Paratyphi A Blood Isolates from Kolkata, India during 2009-2013. PLOS ONE e101347, 9-8:1-13

5. Mohanty S, Renuka K, Sood S, Das BK, Kapil A. Antibiogram pattern and seasonality of Salmonella serotypes in a North Indian tertiary care hospital. Epidemiol Infect. 2006; 134: 961-6. doi: $10.1017 /$ S0950268805005844.

6. Lathi N, Sudarsana J, Pushpa KK. (2004) Epidemic of Salmonella enteric serotype Paratyphi A in Calicut. Calicut Med J;2; e2

7. Bhattacharya SS, Usha D, Choudhury BK (2011) Occurrence \& antibiogram of Salmonella typhi \& S. paratyphi A isolated from Rourkela, Orissa. Indian J Med Res, 133(4): 431-433. PMCID: PMC3103178.

8. Ragini B, Vandana KE, Shivashankara KN, Rohit V, Vishwanath S (2010) Paratyphoid fever- Emerging problem in South India. Asian Pacific Journal of Tropical Medicine, 815-817.

9. Varsha Gupta, Jaspal Kaur \& Jagdish Chander (2009) An increase in enteric fever cases due to Salmonella paratyphi $A$ in \& around Chandigarh. Indian $\mathbf{J}$ Med Res 129: 95-98

10. William RH (1994) Group 5: Facultatively anaerobic Gram negative rods. In Bergey's manual of determinative bacteriology. 9th 270 ed Baltimore: Williams and Wilkins 175201.

11. Ruth MB and Old DC (1989), The usefulness of biotyping in studying the epidemiology and phylogeny of salmonellae. J. Med. Microbiol. 29: 8188.

12. Popoff MY and Le Minor L (1992) Antigenic formulas of the Salmonella serovars. 7th revision. WHO Collaborating Centre for Reference 264 
Research on Salmonella. Paris: Institute Pasteur.

13. Francois XW, Laetitia F, Bernadette G, Patrick AD, Grimont and Isabelle $\mathrm{C}$ (2005) Multiple-Antibiotic Resistance in Salmonella enterica Serotype Paratyphi B Isolates Collected in France between 2000 and 2003 Is Due Mainly to Strains Harboring Salmonella Genomic Islands 1, 1-B, and 1-C. Antimicrobial Agents and Chemotherapy, 2793-2801

14. Verma S, Thakur S, Kanga A, et. al. (2010) Emerging Salmonella paratyphi A enteric fever and changing trends in antimicrobial resistance pattern of Salmonella in Shimla. Indian Journal of Medical Microbiology, 28(1): 51-53

15. Tankhiwale SS, G. Agrawal \& S.V. Jalgaonkar (2003) An unusually high occurrence of Salmonella enterica serotype Paratyphi A in patients with enteric fever. Indian J Med Res, 117: 10-12.

16. Aoife Molloy, Satheesh Nair, Fiona JC, John Wain, Mark Farrington, Paul JL and M. Estee Torok, () First Report of Salmonella enterica Serotype Paratyphi A Azithromycin Resistance Leading to Treatment Failure. Journal Of Clinical Microbiology, 48:12, 4655-4657, 0095$1137 / 10 / \$ 12.00$ doi:10.1128/JCM.00648-10

17. F. Yu \& S. Fan \& X. Fan \& P. Dong \& X. Wang \& Y. Chen \& J. Li (2011) Analysis of characteristics of paratyphoid A in 157 Chinese inpatients between 1998 and 2009 Eur J Clin Microbiol Infect Dis, 30:71-75 DOI

\subsection{7/s10096-010-1055-3}

18. Romney M H, Nava Yeganeh, Kevin WW, Lewinski MA and Natascha Ching, (2011), Enteric Fever in a 6Year-Old Traveler Caused by Salmonella enterica Serotypes Typhi and Paratyphi A: Laboratory Detection Strategies and Treatment Options. Journal Of Clinical Microbiology, 49:1, 452-454. 0095-1137/11/\$12.00 doi:10.1128/JCM.01316-10

19. Orit Gat, James EG, Sharon T, Raphael S, William C. Blackwelder, David JS, Marcela FP, Myron ML (2011) CellAssociated Flagella Enhance the Protection Conferred by MucosallyAdministered Attenuated Salmonella paratyphi A Vaccines. PLOS Neglected Tropical Diseases, 5 :11: e1373: 1-11

20. E.Konadu, J. Shiloach, D.A.Bryla, J.B. Robbins and S. C. Szu, (1996) Synthesis, Characterization and Immunological properties in mice of conjugates composed of detoxified lipopolysacharide of Salmonella paratyphi A bound to tetanus toxoid with emphasis on the role of O-acetyls. Infection and Immunity, 64:7, 27092715

21. Aamir Ali, So J An, Changfa Cui, Abdul Haque and Rodney Carbis, (2014) Synthesis and Immunogenicity evaluation of Salmonella enteric serovar Paratyphi A O-specific polysacharide conjugated to diptheria toxoid. Human vaccines and Immunotherapeutics, 10:6, 1494-98.

\section{How to cite this article:}

Arti Bharmoria, Shahana Majumdar and Vipin Behari Vaish. 2016. Typing and Growth Characteristics of Salmonella paratyphi A obtained from Blood sample of a Paratyphoid Case. Int.J.Curr.Microbiol.App.Sci.5(2): 769-774. doi: http://dx.doi.org/10.20546/ijcmas.2016.502.087 